\title{
TRANSFORMATION OF FAMILIES OF MATRICES TO NORMAL FORMS AND ITS APPLICATION TO STABILITY THEORY*
}

\author{
ALEXEI A. MAILYBAEV ${ }^{\dagger}$
}

\begin{abstract}
Families of matrices smoothly depending on a vector of parameters are considered. Arnold [Russian Math. Surveys, 26 (1971), pp. 29-43] and Galin [Uspekhi Mat. Nauk, 27 (1972), pp. 241-242] have found and listed normal forms of families of complex and real matrices (miniversal deformations), to which any family of matrices can be transformed in the vicinity of a point in the parameter space by a change of basis, smoothly dependent on a vector of parameters, and by a smooth change of parameters. In this paper a constructive method of determining functions describing a change of basis and a change of parameters, transforming an arbitrary family to the miniversal deformation, is suggested. Derivatives of these functions with respect to parameters are determined from a recurrent procedure using derivatives of the functions of lower orders and derivatives of the family of matrices. Then the functions are found as Taylor series. Examples are given. The suggested method allows using efficiently miniversal deformations for investigation of different properties of matrix families. This is shown in the paper where tangent cones (linear approximations) to the stability domain at the singular boundary points are found.
\end{abstract}

Key words. family of matrices, normal form, versal deformation, stability, tangent cone

AMS subject classifications. 15A21, 15A90, 58F36

PII. S0895479898338378

1. Introduction. The paper deals with the study of families of complex and real matrices (a family of matrices is a matrix-valued function $A(p)$ holomorphically (smoothly) depending on a vector $p$ of complex (real) parameters). It is known that from all families of matrices we can select such families that transformation of any family to them can be carried out by a change of basis, smoothly depending on parameters, and by a smooth change of parameters $[1,2,3]$. Such families are called versal deformations. Versal deformations with the minimum possible number of parameters are called miniversal deformations or normal forms. Miniversal deformations of complex matrices have been found by Arnold $[1,2,3]$. He showed that the miniversal deformation, to which a family of matrices $A(p)$ can be transformed in the vicinity of a point $p=p_{0}$, is determined by the Jordan form of the matrix $A\left(p_{0}\right)$. Miniversal deformations of real matrices have been studied by Galin [8].

to .5pt The important part of the transformation of a family of matrices to the normal form is to find functions describing a change of basis, depending on parameters, and a change of parameters. Determination of these functions is necessary for effective use of normal forms, but no general algorithm for finding them is available yet. Efforts of construction of the transformation functions required in a specific case have been done in $[5,12]$. In the case when the matrix $A_{0}=A\left(p_{0}\right)$ has only nonderogatory eigenvalues, the problem of construction of transformation to the normal form has been discussed in [13]. However, the method of finding the transformation functions suggested in [13] can be used only for matrices of small dimensions or in the case when the matrix $A_{0}$ has only one nonderogatory eigenvalue. Otherwise it leads to a

\footnotetext{
${ }^{*}$ Received by the editors May 6, 1998; accepted for publication (in revised form) by M. Overton February 22, 1999; published electronically November 2, 1999.

http://www.siam.org/journals/simax/21-2/33837.html

$\dagger$ Institute of Mechanics, Moscow State Lomonosov University, Michurinsky pr. 1, 117192 Moscow, Russia (mailybaev@inmech.msu.su).
} 
complicated system of nonlinear equations containing parameters of the family and parameters of the normal form.

Another approach has been suggested in [14], where the transformation functions have been found at a specific value of the parameter vector $p$ by solving a nonlinear implicit matrix equation using secant, updating secant, or using Newton's method. Such a numerical approach, though useful for finding functions in a finite domain (functions are calculated at several points and then approximated), is not efficient in the case under consideration. This is connected with the local character of miniversal deformations, which are defined in the vicinity of a point in the parameter space and used for investigation of local properties of matrix families (singularities, bifurcations, etc.). For local analysis, information about derivatives of the transformation functions with respect to parameters is required. Computation of these derivatives by means of approximate methods of [14] is very time-consuming and leads to numerical errors (especially when the size of the matrix $A$ is not small). In [14] also a method for computation of derivatives of the transformation functions for a case of one-parameter matrix family has been suggested. This family had a special form allowing transformation to a normal form which was simpler than a miniversal deformation.

In the present paper a constructive method of finding functions describing the transformation of any given family of matrices to the normal form is suggested. The functions are found as Taylor series. Derivatives of the functions with respect to parameters, to determine coefficients of Taylor series, are found from a recurrent procedure using the derivatives of lower orders of these functions and derivatives of the family at the initial value of the vector of parameters. The recurrent procedure is convenient for numerical implementation, since it consists of elementary arithmetic operations. Proofs are based on properties of centralizers of matrices transformed to the Jordan form.

Transformation of a family of matrices to the normal form has great significance for applications. It allows studying specific families (normal forms), which consist usually of sparse matrices depending on parameters in a simple way, and then obtaining results for arbitrary families of matrices. Miniversal deformations (without knowledge about transformation to them) have been used in $[1,2,3]$ for classification of singularities of bifurcation diagrams, decrement diagrams, and boundaries of the stability domains in the case of two and three parameters. In [10] miniversal deformations have been used for finding tangent cones (linear approximations) to the stability domain at singular boundary points up to a diffeomorphism. All previous results were qualitative. To obtain quantitative results we need information about the transformation. In [4] specific linear combinations of first derivatives of the functions describing a change of parameters have been found and used to obtain necessary conditions for the stable perturbations of matrices. Other applications of miniversal deformations can be found in [6, 7], where a bound for a distance to a less generic matrix pencil and backward error bound in the procedure of calculation of polynomial roots are obtained.

The method of finding functions of transformation, suggested in this paper, allows using miniversal deformations for quantitative study of different properties of matrices depending on parameters. Efficiency of the method for applications is illustrated by the problem of finding tangent cones (or parts of tangent cones) to the stability domain for singular boundary points. All types of singular boundary points are considered. The obtained results represent an extension of the paper [11], where, using another approach, tangent cones for singular boundary points of the stability domain of twoand three-parameter generic families of matrices have been found. 
The paper is organized as follows. In section 2 the method for determining derivatives of functions describing the transformation of a family of complex matrices to miniversal deformation is formulated and proved. Section 3 discusses the case of families of real matrices. Section 4 uses results of the previous sections to find tangent cones to the stability domain at singular boundary points. The conclusion gives a brief overview of the results obtained and discusses possibilities for their application to different problems.

2. Transformation of families of complex matrices to normal forms. For the following presentation we need definitions given by Arnold [1, 2], [3, p. 237].

Definition 2.1. A family of complex matrices is a holomorphic mapping A : $P \longrightarrow \mathbf{C}^{n^{2}}$, where $P$ is the vicinity of some point $p_{0}$ in the parameter space $\mathbf{C}^{m}$ and $\mathbf{C}^{n^{2}}$ is the space of $n \times n$ complex matrices.

Let us consider an arbitrary family of matrices $A(p), p=\left(p_{1}, \ldots, p_{m}\right)^{T} \in \mathbf{C}^{m}$. Denote by $\lambda_{i}$ the eigenvalues of the matrix $A_{0}=A\left(p_{0}\right)$ and let $n_{1}\left(\lambda_{i}\right) \geq n_{2}\left(\lambda_{i}\right) \geq \cdots$ be the dimensions of the Jordan blocks belonging to $\lambda_{i}$, beginning with the largest one. Let the Jordan form $J$ of the matrix $A_{0}$ be given by

$$
A_{0}=C_{0} J C_{0}^{-1},
$$

where

$$
\begin{gathered}
J=\left(\begin{array}{lll}
J_{1} & & \\
& J_{2} & \\
& & \ddots
\end{array}\right), \\
J_{i}=\left(\begin{array}{lll}
J_{i}^{1} & & \\
& J_{i}^{2} & \\
& & \ddots
\end{array}\right),
\end{gathered}
$$

and the Jordan block

$$
J_{i}^{k}=\left(\begin{array}{ccccc}
\lambda_{i} & 1 & & & \\
& \cdot & \cdot & & \\
& & \cdot & \cdot & \\
& & & \cdot & 1 \\
& & & & \lambda_{i}
\end{array}\right)
$$

has dimension $n_{k}\left(\lambda_{i}\right)$.

Denote by $X_{i j}^{k l}$ the block of a square $n \times n$ matrix $X$, which is situated on the intersection of the rows corresponding to the block $J_{i}^{k}$ and columns corresponding to the block $J_{j}^{l}$; see, for example, Figure 2.1. Denote elements of each block $X_{i j}^{k l}$ by $x_{i j}^{k l}(r, s), r=1, \ldots, n_{k}\left(\lambda_{i}\right), s=1, \ldots, n_{l}\left(\lambda_{j}\right)$, where $r, s$ are the numbers of a row and a column in $X_{i j}^{k l}$ corresponding to $x_{i j}^{k l}(r, s)$. In the case $i=j$ we will leave only one number in the subscript, e.g., $X_{i}^{k l}, x_{i}^{k l}(r, s)$. The block of the matrix $X$ which corresponds to the block $J_{i}$ of the Jordan form $J$ will be denoted by $X_{i}$.

Definition 2.2. A versal deformation of a matrix $A_{0}$ is a family of matrices $A^{\prime}\left(p^{\prime}\right), p^{\prime} \in \mathbf{C}^{d}$ such that any family $A(p), A\left(p_{0}\right)=A_{0}$ in the vicinity of the point $p=p_{0}$ can be represented in the form

$$
A(p)=C(p) A^{\prime}(\varphi(p)) C^{-1}(p),
$$




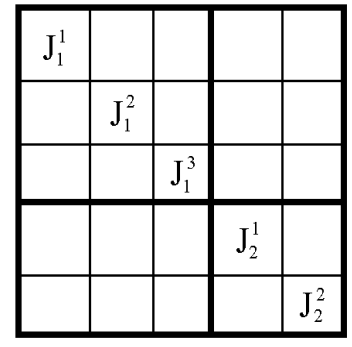

(a)

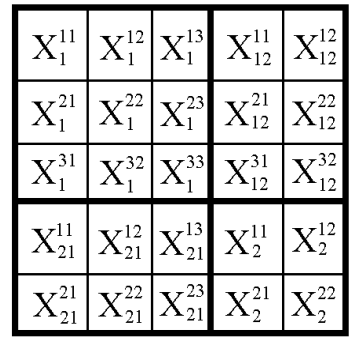

(b)

FIG. 2.1. Block decomposition of a square matrix $X$ according to the Jordan form $J$.

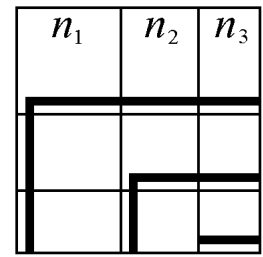

(a)

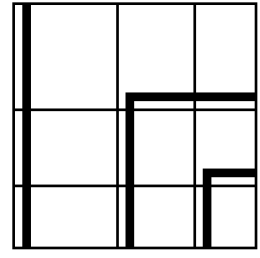

(b)

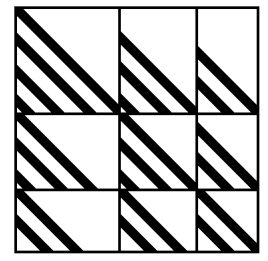

(c)

FIG. 2.2. Location of parameters in blocks of a miniversal deformation.

where $p^{\prime}=\varphi(p), \varphi\left(p_{0}\right)=\mathbf{0}$ is a holomorphic mapping from the vicinity of the point $p=p_{0}$ in the parameter space $\mathbf{C}^{m}$ into the vicinity of the origin of coordinate in the space $\mathbf{C}^{d} ; C(p)$ is a family of nonsingular matrices describing a change of basis. A versal deformation is said to be miniversal if the dimension d of the parameter space is the smallest possible for a versal deformation [1, 2], [3, p. 237].

It is shown in [1, 2], [3, p. 238], that a miniversal deformation is determined by the Jordan form of the matrix $A_{0}$ and can be chosen in the form of a sum $J+B\left(p^{\prime}\right)$, where $B\left(p^{\prime}\right)=\operatorname{Diag}\left(B_{1}, B_{2}, \ldots\right)$ is a family of block-diagonal matrices with blocks $B_{i}\left(p^{\prime}\right)$ corresponding to the blocks $J_{i}$ of the matrix $J$. There are different ways of choosing the blocks $B_{i}\left(p^{\prime}\right)$. Three possible types are shown in Figure 2.2, where the block $B_{i}\left(p^{\prime}\right)$ has all zeros except at the places indicated. In cases (a) and (b), the indicated places are filled by different components of the vector $p^{\prime}$ (parameters of the miniversal deformation); in case (c) each slanted segment is filled by a corresponding component of the vector $p^{\prime}$. Other types of blocks are also possible, e.g., blocks whose elements are all zero, except for one element on each slanted segment in Figure 2.2(c) which is an independent parameter. In all cases, each block $B_{i}^{k l}$ contains exactly $n_{l}\left(\lambda_{i}\right)$, if $k \leq l$, and $n_{k}\left(\lambda_{i}\right)$, if $k>l$, different components of $p^{\prime}$ (one on each slanted segment in Figure 2.2(c)). Denote these components by $b_{i}^{k l}(t), t=1, \ldots, \min \left(n_{k}\left(\lambda_{i}\right), n_{l}\left(\lambda_{i}\right)\right)$ numbered inside each block from the top and from the right (see examples for the case of two Jordan blocks of the size 3 and 2 in Figure 2.3). Components $b_{i}^{k l}(t)$ compose the vector of parameters of the miniversal deformation $p^{\prime}$ of the dimension $d=\sum_{i}\left(n_{1}\left(\lambda_{i}\right)+3 n_{2}\left(\lambda_{i}\right)+\cdots\right)[1,2],[3$, p. 237].

Definition 2.3. Define a generalized trace of a $k \times l$ matrix $F$ denoted by $\operatorname{tr}^{(t)} F$ as a sum of elements on that diagonal of $F$ which is $(\min (k, l)-t)$ positions above the left lower element 


\begin{tabular}{|c|c|c|c|c|}
\hline 0 & 0 & 0 & 0 & 0 \\
\hline 0 & 0 & 0 & 0 & 0 \\
\hline$b_{i}^{11}(3)$ & $b_{i}^{11}(2)$ & $b_{i}^{11}(1)$ & $b_{i}^{12}(2)$ & $b_{i}^{12}(1)$ \\
\hline$b_{i}^{21}(1)$ & 0 & 0 & 0 & 0 \\
\hline$b_{i}^{21}(2)$ & 0 & 0 & $b_{i}^{22}(2)$ & $b_{i}^{22}(1)$ \\
\hline
\end{tabular}

(a)

\begin{tabular}{|c|c|c|c|c|}
\hline$b_{i}^{11}(1)$ & 0 & 0 & 0 & 0 \\
\hline$b_{i}^{11}(2)$ & 0 & 0 & 0 & 0 \\
\hline$b_{i}^{11}(3)$ & 0 & 0 & $b_{i}^{12}(2)$ & $b_{i}^{12}(1)$ \\
\hline$b_{i}^{21}(1)$ & 0 & 0 & $b_{i}^{22}(1)$ & 0 \\
\hline$b_{i}^{21}(2)$ & 0 & 0 & $b_{i}^{22}(2)$ & 0 \\
\hline
\end{tabular}

(b)

\begin{tabular}{|c|c|c|c|c|}
\hline$b_{i}^{11}(1)$ & 0 & 0 & 0 & 0 \\
\hline$b_{i}^{11}(2)$ & $b_{i}^{11}(1)$ & 0 & $b_{i}^{12}(1)$ & 0 \\
\hline$b_{i}^{11}(3)$ & $b_{i}^{11}(2)$ & $b_{i}^{11}(1)$ & $b_{i}^{12}(2)$ & $b_{i}^{12}(1)$ \\
\hline$b_{i}^{21}(1)$ & 0 & 0 & $b_{i}^{22}(1)$ & 0 \\
\hline$b_{i}^{21}(2)$ & $b_{i}^{21}(1)$ & 0 & $b_{i}^{22}(2)$ & $b_{i}^{22}(1)$ \\
\hline
\end{tabular}

(c)

FIG. 2.3. Numbering of parameters of a miniversal deformation.

$$
\operatorname{tr}^{(t)} F=\sum_{r=t}^{\min (k, l)} f(k-\min (k, l)+r, r-t+1) .
$$

In the case of a square matrix $(k=l)$, Definition 2.3 coincides with the definition of a generalized trace given in [4]. In particular, a generalized trace $\operatorname{tr}^{(\min (k, l))} F$ is equal to the left lower element of the matrix $F$, and a generalized $\operatorname{trace} \operatorname{tr}^{(1)} F$ of a square matrix $F$ coincides with the ordinary trace $\operatorname{tr} F$, which is equal to the sum of elements on the main diagonal. In the case of an $n \times n$ matrix $F$ a generalized trace $\operatorname{tr}^{(t)}$ of a block $F_{i}^{k l}$ is equal to the sum of elements on that slanted segment which is $(t-1)$ positions below the longest one in this block; see Figure 2.2,

$$
\operatorname{tr}^{(t)} F_{i}^{k l}=\sum_{r=t}^{n^{\prime}} f_{i}^{k l}\left(n_{k}\left(\lambda_{i}\right)-n^{\prime}+r, r-t+1\right), \quad n^{\prime}=\min \left(n_{k}\left(\lambda_{i}\right), n_{l}\left(\lambda_{i}\right)\right) .
$$

For blocks of the matrix $B\left(p^{\prime}\right)$ we have $\operatorname{tr}^{(t)} B_{i}^{k l}=\alpha_{i}^{k l}(t) b_{i}^{k l}(t)$, where $\alpha_{i}^{k l}(t)=1$, if $B_{i}$ is a block of the type (a) or (b), and $\alpha_{i}^{k l}(t)=n^{\prime}-t+1$, if $B_{i}$ is a block of the type (c); see Figure 2.2.

Denote by $D^{h}$ the derivative

$$
D^{h}=\frac{\partial^{|h|}}{\partial p_{1}^{h^{1}} \cdots \partial p_{m}^{h^{m}}}
$$

taken at the point $p=p_{0}$, where $h=\left(h^{1}, \ldots, h^{m}\right), h^{i} \geq 0$, is a vector whose components $h^{i}$ denote the order of the partial derivative with respect to $p_{i}\left(h^{i}=0\right.$ means that the derivative with respect to $p_{i}$ is not taken), $|h|=h^{1}+\cdots+h^{m}$ is the order of the derivative.

Transformation of the family of matrices $A(p)$ to the normal form (miniversal deformation) (2.2) includes finding the family $A^{\prime}\left(p^{\prime}\right)$ corresponding to the Jordan form $J$ of the matrix $A_{0}$, families $C(p), C^{-1}(p)$, and holomorphic mapping $p^{\prime}=$ $\varphi(p)$ (functions $b_{i}^{k l}(t)(p)$ ). To obtain $C(p), C^{-1}(p)$, and $b_{i}^{k l}(t)(p)$, it is sufficient to determine derivatives $D^{h} C, D^{h} C^{-1}$, and $D^{h} b_{i}^{k l}(t)$ for all $h$. Then the desired functions are found locally as Taylor series (by virtue of holomorphy).

THEOREM 2.4. Let $A(p)$ be a family of matrices. Then the following expressions are valid for derivatives $D^{h},|h|>0$ of the functions $C(p), C^{-1}(p), b_{i}^{k l}(t)(p)$ transforming the family of matrices $A(p)$ to the miniversal deformation $A^{\prime}\left(p^{\prime}\right)$ described above (2.2). 
$1^{\circ}$

$$
\begin{gathered}
D^{h} b_{i}^{k l}(t)=\frac{\operatorname{tr}^{(t)} F_{i}^{k l}}{\alpha_{i}^{k l}(t)}, \\
F=C_{0}^{-1}\left(D^{h} A-\sum_{\substack{h_{1}+h_{2}+h_{3}=h \\
h_{1}, h_{2}, h_{3} \neq h}} C_{h}^{h_{1} h_{2} h_{3}} D^{h_{1}} C D^{h_{2}} A^{\prime} D^{h_{3}} C^{-1}\right. \\
\left.+A_{0} \sum_{\substack{h_{1}+h_{2}=h \\
h_{1}, h_{2} \neq h}} C_{h}^{h_{1} h_{2}} D^{h_{1}} C D^{h_{2}} C^{-1}\right) C_{0}, \\
C_{h}^{h_{1} h_{2} h_{3}}=\prod_{i=1}^{m} \frac{h^{i} !}{h_{1}^{i} ! h_{2}^{i} ! h_{3}^{i} !}, \quad C_{h}^{h_{1} h_{2}}=\prod_{i=1}^{m} \frac{h^{i} !}{h_{1}^{i} ! h_{2}^{i} !} .
\end{gathered}
$$

$2^{\circ}$.

$x_{i j}^{k l}(r, s)=\sum_{r_{1}=r}^{n_{k}\left(\lambda_{i}\right)} \sum_{s_{1}=1}^{s} \frac{(-1)^{r_{1}-r}}{\left(\lambda_{i}-\lambda_{j}\right)^{r_{1}-r+s-s_{1}+1}} C_{r_{1}-r+s-s_{1}}^{r_{1}-r} g_{i j}^{k l}\left(r_{1}, s_{1}\right), i \neq j$,

$$
\begin{aligned}
& C_{n}^{k}=\frac{n !}{k !(n-k) !}, \\
& x_{i}^{k l}(r, s)=\left\{\begin{array}{cl}
0, & k \leq l, r=1, \\
\sum_{s_{1}=s^{\prime}(r, s)}^{s} g_{i}^{k l}\left(r-s+s_{1}-1, s_{1}\right), & k \leq l, r \neq 1, \\
-\sum_{r_{1}=r}^{r^{\prime}(r, s)} g_{i}^{k l}\left(r_{1}, s-r+r_{1}+1\right), & k>l, s \neq n_{l}\left(\lambda_{i}\right), \\
0, & k>l, s=n_{l}\left(\lambda_{i}\right),
\end{array}\right. \\
& s^{\prime}(r, s)=\max (1, s-r+2), \quad r^{\prime}(r, s)=\min \left(n_{k}\left(\lambda_{i}\right), r-s+n_{l}\left(\lambda_{i}\right)-1\right), \\
& G=D^{h} A^{\prime}-F .
\end{aligned}
$$

$3^{\circ}$.

$$
D^{h} C^{-1}=-C_{0}^{-1} \sum_{\substack{h_{1}+h_{2}=h \\ h_{2} \neq h}} C_{h}^{h_{1} h_{2}} D^{h_{1}} C D^{h_{2}} C^{-1} .
$$

The zero order derivatives $D^{h}, h=\mathbf{0}$ of functions $C, C^{-1}, A^{\prime}$ (values of functions at $\left.p=p_{0}\right)$ are $D^{0} C=C_{0}, D^{0} C^{-1}=C_{0}^{-1}, D^{0} A^{\prime}=J$. Theorem 2.4 allows finding 
derivatives $D^{h}$ of the order $|h|>0$ of functions $C, C^{-1}$, and $b_{i}^{k l}(t)$, using only derivatives of these functions of a lower order and the derivative $D^{h} A$. Thus, (2.4)-(2.10) represent a recurrent procedure for determining the derivatives $D^{h} C, D^{h} C^{-1}$, and $D^{h} b_{i}^{k l}(t)$ for any $h$ using derivatives $D^{h_{1}} A$ of the order $\left|h_{1}\right|<|h|$ and $h_{1}=h$.

If all derivatives $D^{h} C, D^{h} C^{-1}$, and $D^{h} b_{i}^{k l}(t)$ of the order $|h| \leq s$ are determined, the desired functions can be found approximately as Taylor series containing terms with derivatives $D^{h}$ of the order $|h| \leq s$

$$
\begin{gathered}
C(p)=\sum_{0 \leq|h| \leq s} D^{h} C \prod_{r=1}^{m} \frac{\left(p_{r}-p_{0 r}\right)^{h^{r}}}{h^{r} !}+o\left(\left\|p-p_{0}\right\|^{s}\right), \\
C^{-1}(p)=\sum_{0 \leq|h| \leq s} D^{h} C^{-1} \prod_{r=1}^{m} \frac{\left(p_{r}-p_{0 r}\right)^{h^{r}}}{h^{r} !}+o\left(\left\|p-p_{0}\right\|^{s}\right), \\
b_{i}^{k l}(t)(p)=\sum_{0 \leq|h| \leq s} D^{h} b_{i}^{k l}(t) \prod_{r=1}^{m} \frac{\left(p_{r}-p_{0 r}\right)^{h^{r}}}{h^{r} !}+o\left(\left\|p-p_{0}\right\|^{s}\right) .
\end{gathered}
$$

Here $\|p\|$ is the norm in $\mathbf{C}^{m}$. Denoting these approximations by $\widetilde{C}(p), \widetilde{C}^{-1}(p)$, and $\widetilde{\varphi}(p)$ we can represent the family of matrices $A(p)$ in the form $A(p)=\widetilde{C}(p) A^{\prime}(\widetilde{\varphi}(p)) \widetilde{C}^{-1}(p)+$ $o\left(\left\|p-p_{0}\right\|^{s}\right)$; that is, the recurrent procedure allows transforming an arbitrary given family of matrices $A(p)$ to the normal form in the vicinity of the point $p=p_{0}$ with the accuracy up to small terms of an arbitrary order.

Note that Burke and Overton [4] obtained the formulae for calculation specific sums of the first order derivatives of functions $b_{i}^{k l}(t)(p)$ in the case of one parameter $p \in \mathbf{R}$. These formulae can be derived from expressions (2.4) and (2.5).

2.1. Example. As an example let us consider a one-parameter family of $3 \times 3$ matrices $A(z)$ which has at the point $z=0$ eigenvalues $\lambda_{1}=0, \lambda_{2}=2 i$ with corresponding Jordan blocks of the order $n_{1}\left(\lambda_{1}\right)=2, n_{1}\left(\lambda_{2}\right)=1$, respectively,

$$
A(z)=\left(\begin{array}{ccc}
2 i+z & -8 z & z^{2} \\
2 i z & 4 i z & 1-i z^{2} \\
2 z^{2} & -2 z & z^{2}
\end{array}\right) .
$$

The Jordan form of the matrix $A_{0}=A(0)$ and matrices $C_{0}, C_{0}^{-1}$ have the form

$$
J=\left(\begin{array}{ccc}
0 & 1 & 0 \\
0 & 0 & 0 \\
0 & 0 & 2 i
\end{array}\right), \quad C_{0}=\left(\begin{array}{ccc}
0 & 0 & 1 \\
1 & 0 & 0 \\
0 & 1 & 0
\end{array}\right), \quad C_{0}^{-1}=\left(\begin{array}{ccc}
0 & 1 & 0 \\
0 & 0 & 1 \\
1 & 0 & 0
\end{array}\right) .
$$

For the first iteration $\left(h=1, D^{h}=d / d z\right)$ we have

$$
\begin{gathered}
F=\left(\begin{array}{ccc}
f_{1}^{11}(1,1) & f_{1}^{11}(1,2) & f_{12}^{11}(1,1) \\
f_{1}^{11}(2,1) & f_{1}^{11}(2,2) & f_{12}^{11}(2,1) \\
f_{21}^{11}(1,1) & f_{21}^{11}(1,2) & f_{2}^{11}(1,1)
\end{array}\right)=\left(\begin{array}{ccc}
4 i & 0 & 2 i \\
-2 & 0 & 0 \\
-8 & 0 & 1
\end{array}\right), \\
G=\left(\begin{array}{ccc}
-4 i & 0 & -2 i \\
0 & 4 i & 0 \\
8 & 0 & 0
\end{array}\right), \quad X=\left(\begin{array}{ccc}
0 & 0 & 1 \\
-4 i & 0 & 0 \\
-4 i & -2 & 0
\end{array}\right) .
\end{gathered}
$$




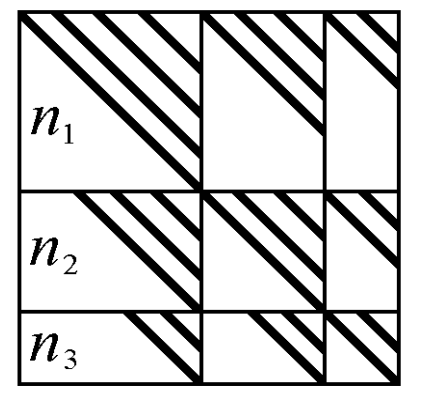

FIG. 2.4. Block of a matrix commuting with the Jordan form J.

These matrices are used in (2.4), (2.6), and (2.10) to determine the first derivatives of the desired functions. Then these derivatives are used in the second iteration to find the derivatives $D^{h}=d^{2} / d z^{2}$. As a result, the normal form $A^{\prime}\left(p^{\prime}\right)$ of the family $A(z)$ and, determined by Theorem 2.4, the functions $p^{\prime}=\varphi(z), C(z), C^{-1}(z)$ have the form

$$
\begin{aligned}
& A^{\prime}\left(p^{\prime}\right)=\left(\begin{array}{ccc}
0 & 1 & 0 \\
b_{1}(2) & b_{1}(1) & 0 \\
0 & 0 & 2 i+b_{2}(1)
\end{array}\right), \quad p^{\prime}=\left(b_{1}(1), b_{1}(2), b_{2}(1)\right)^{T}, \\
& b_{1}(1)=4 i z+9 z^{2}+o\left(z^{2}\right), \quad b_{1}(2)=-2 z+o\left(z^{2}\right), \quad b_{2}(1)=z-8 z^{2}+o\left(z^{2}\right), \\
& C(z)=\left(\begin{array}{ccc}
-4 i z+(2-2 i) z^{2} & -2 z-(5+1.5 i) z^{2} & 1 \\
1 & 0 & z+(2+0.5 i) z^{2} \\
-4 i z-8 z^{2} & 1+5 i z^{2} & 0
\end{array}\right)+o\left(z^{2}\right) \\
& C^{-1}(z)=\left(\begin{array}{ccc}
-z-(2+0.5 i) z^{2} & 1-4 i z^{2} & -2 z^{2} \\
-4 i z^{2} & 4 i z+8 z^{2} & 1-5 i z^{2} \\
1-4 i z^{2} & 4 i z-(2-10 i) z^{2} & 2 z+(5+1.5 i) z^{2}
\end{array}\right)+o\left(z^{2}\right) .
\end{aligned}
$$

\subsection{Proof of the theorem.}

Definition 2.5. The centralizer of a square matrix $X$ is the set of all matrices commuting with $X$. The notation is as follows:

$$
Z_{X}=\{Y:[X, Y]=0\},
$$

where $[X, Y]=X Y-Y X[1,2,3]$.

A centralizer $Z_{J}$ of the Jordan form $J$ consists of block-diagonal matrices $Y=$ $\operatorname{Diag}\left(Y_{1}, Y_{2}, \ldots\right)$ whose blocks $Y_{i}$ correspond to the blocks $J_{i}$ and have the form shown in Figure $2.4[1,2,3,7]$. In Figure 2.4 every slanted segment denotes a sequence of equal numbers, while other elements are all zeros. The centralizer $Z_{J}$ is a plane in the space of all matrices $\mathbf{C}^{n^{2}}$ which passes through the zero and identity matrix.

Lemma 2.6. For any $n \times n$ matrix $X$ and any $i, k, l, t$ the following equality is valid:

$$
\operatorname{tr}^{(t)}\left([J, X]_{i}^{k l}\right)=0 .
$$


Proof. For any matrix $Y \in Z_{J}$ we have

$$
\begin{aligned}
& \operatorname{tr}([J, X] Y)=\operatorname{tr}(J X Y-X J Y)= \\
& \operatorname{tr}(Y J X-J Y X)=-\operatorname{tr}([J, Y] X)=0 .
\end{aligned}
$$

Consider the matrix $Y$ which has units on the $t$ th slanted segment (beginning from the largest one) in the block $Y_{i}^{l k}$ (see Figure 2.4), while the other components are all zeros. Then, using (2.11), we obtain $0=\operatorname{tr}([J, X] Y)=\operatorname{tr}^{(t)}\left([J, X]_{i}^{k l}\right)$.

Lemma 2.7. For any $n \times n$ matrix $G$, which satisfies the condition $\operatorname{tr}^{(t)} G_{i}^{k l}=0$ for all $i, k, l, t$, the equation

$$
[J, X]=G
$$

has the unique solution (2.7), (2.8), satisfying

$$
\begin{array}{ll}
x_{i}^{k l}(1, s)=0, & k \leq l, \\
x_{i}^{k l}\left(s, n_{l}\left(\lambda_{i}\right)\right)=0, & k>l,
\end{array}
$$

for all $i, k, l, s$.

Proof. In the case when $G$ is the zero matrix a set of solutions of (2.12) is the centralizer $Z_{J}$. To satisfy conditions (2.13) the matrix $X \in Z_{J}$ must have zeros on the sth slanted segment (beginning from the smallest one for $k \leq l$ and from the largest one for $k>l$ ) of the block $X_{i}^{k l}$; see Figure 2.4. Hence, the equation $[J, X]=0$ has the unique solution $X=0$ satisfying all conditions (2.13). Due to the linearity of equation (2.12) and conditions (2.13), if two matrices $X_{1}$ and $X_{2}$ are solutions of (2.12) and (2.13) for nonzero matrix $G$, then the matrix $X=X_{1}-X_{2}$ satisfies the equations $[J, X]=0$ and (2.13). Consequently, $X=X_{1}-X_{2}=0$; that is, if a solution of (2.12) and (2.13) exists, it is unique.

By substituting the explicit form of $J$ into (2.12) we obtain equations on the elements of the matrix $X$

$$
\left(\lambda_{i}-\lambda_{j}\right) x_{i j}^{k l}(r, s)+x_{i j}^{k l}(r+1, s)-x_{i j}^{k l}(r, s-1)=g_{i j}^{k l}(r, s),
$$

where, instead of all elements $x_{i j}^{k l}$ with coordinates $(r+1, s)$ or $(r, s-1)$, which don't belong to the block $X_{i j}^{k l}$, we take zeros. The fact that (2.7) and (2.8) is a solution to equations (2.12) and (2.13) can be verified by substitution of expressions (2.7) and (2.8) into (2.13) and (2.14). After substitution, equations (2.14) for $i=j, r=n_{k}\left(\lambda_{i}\right)$, $s=n_{l}\left(\lambda_{i}\right)-t+1, k \leq l$ and for $i=j, r=t, s=1, k>l$ take the form $\operatorname{tr}^{(t)} G_{i}^{k l}=0$. They are satisfied by virtue of the conditions of the lemma. Other equations are satisfied identically.

Proof of Theorem 2.4. The proof is divided into three parts according to the items of the theorem. Each part is denoted by a corresponding item number.

Item $3^{\circ}$. Let us take the derivative $D^{h},|h|>0$ from both sides of the equality $C(p) C^{-1}(p)=I$, where $I$ is the identity matrix:

$$
\sum_{h_{1}+h_{2}=h} C_{h}^{h_{1} h_{2}} D^{h_{1}} C D^{h_{2}} C^{-1}=0, \quad C_{h}^{h_{1} h_{2}}=\prod_{i=1}^{m} \frac{h^{i} !}{h_{1}^{i} ! h_{2}^{i} !} .
$$

After expressing $C_{0} D^{h} C^{-1}$ in terms of the other summands and multiplying both sides of the equation from the left by $C_{0}^{-1}$, we will prove item $3^{\circ}$ of the theorem. 
Item $1^{\circ}$. Let us take the derivative $D^{h}$ from both sides of expression (2.2)

$$
D^{h} A=\sum_{h_{1}+h_{2}+h_{3}=h} C_{h}^{h_{1} h_{2} h_{3}} D^{h_{1}} C D^{h_{2}} A^{\prime} D^{h_{3}} C^{-1}, \quad C_{h}^{h_{1} h_{2} h_{3}}=\prod_{i=1}^{m} \frac{h^{i} !}{h_{1}^{i} ! h_{2}^{i} ! h_{3}^{i} !} .
$$

Expressing $C_{0} D^{h} A^{\prime} C_{0}^{-1}$ in terms of other summands and then multiplying both sides of the equation by $C_{0}^{-1}$ and $C_{0}$ from left and from right, respectively, we obtain

$$
D^{h} A^{\prime}=C_{0}^{-1} D^{h} A C_{0}-C_{0}^{-1} \sum_{\substack{h_{1}+h_{2}+h_{3}=h \\ h_{2} \neq h}} C_{h}^{h_{1} h_{2} h_{3}} D^{h_{1}} C D^{h_{2}} A^{\prime} D^{h_{3}} C^{-1} C_{0} .
$$

Using equation (2.10), proved above for the term containing $D^{h} C^{-1}$, we get

$$
D^{h} A^{\prime}=\left[J, C_{0}^{-1} D^{h} C\right]+F,
$$

where the matrix $F$ is defined in (2.5). Using Lemma 2.6 and taking into account that $\operatorname{tr}^{(t)}\left(D^{h} A_{i}^{\prime k l}\right)=\operatorname{tr}^{(t)}\left(D^{h} B_{i}^{k l}\right)=\alpha_{i}^{k l}(t) D^{h} b_{i}^{k l}(t)$ for $|h|>0$, we will prove item $1^{\circ}$ of the theorem.

Item $2^{\circ}$. Rearranging $(2.15)$, we obtain

$$
\left[J, C_{0}^{-1} D^{h} C\right]=G
$$

where a matrix $G$ is defined in (2.9).

Note that $C_{0}^{-1} D^{h} C=D^{h}\left(C_{0}^{-1} C\right)$ is a derivative of the family of matrices $C_{J}=$ $C_{0}^{-1} C, C_{J}\left(p_{0}\right)=I$, which describe a change of basis transforming the family $A_{J}(p)=$ $C_{0}^{-1} A(p) C_{0}$ to the normal form, that is,

$$
A_{J}(p)=C_{0}^{-1} A(p) C_{0}=C_{0}^{-1} C(p) A^{\prime}(\varphi(p)) C^{-1}(p) C_{0}=C_{J}(p) A^{\prime}(\varphi(p)) C_{J}^{-1}(p) .
$$

At the point $p=p_{0}$ the matrix $A_{J}\left(p_{0}\right)=C_{0}^{-1} A_{0} C_{0}=J$ is in the Jordan form. In the proof of the versality theorem $[1,2]$, [3, pp. 238-241] it was shown that in this case the family $C_{J}$ can be chosen lying on an arbitrary smooth surface of the dimension $n^{2}-d$ in the space of all matrices which passes through the identity matrix and transversal at this point to the centralizer $Z_{J}$. For each such a surface the family $C_{J}$ can be chosen in the unique way. In particular, the plane $I+\Pi$, where $\Pi$ is the set of all matrices $X$ satisfying conditions (2.13), has all these properties. The planes $Z_{J}$ and $I+\Pi$ are transversal, because the only matrix from $Z_{J}$ which satisfies conditions (2.13) (intersection of the planes) is the identity matrix and the sum of dimensions of the planes is equal to the dimension of the space $n^{2}$. If we choose the plane $I+\Pi$ for determining $C_{J}$, we obtain that all derivatives $D^{h} C_{J}=C_{0}^{-1} D^{h} C=X$ must satisfy conditions (2.13), which can be regarded as normality conditions. Then the formulae of item $2^{\circ}$ follow from Lemma 2.7 applied to (2.16) (conditions $\operatorname{tr}^{(t)} G_{i}^{k l}=$ $\operatorname{tr}^{(t)}\left(D^{h} A_{i}^{\prime k l}\right)-\operatorname{tr}^{(t)} F_{i}^{k l}=\alpha_{i}^{k l}(t) D^{h} b_{i}^{k l}(t)-\operatorname{tr}^{(t)} F_{i}^{k l}=0$ are fulfilled by virtue of equality $(2.4))$.

Note that instead of normality conditions (2.13) one can take the same amount of any other linearly independent homogeneous equations such that the only matrix from $Z_{J}$ which satisfies them is the zero matrix. Theorem 2.4 and Lemma 2.7 will remain valid if (2.8) is changed in accordance with the new normality conditions by solving (2.14) for $i=j$, which determines elements of the blocks $X_{i}^{k l}$.

The case of families of complex matrices $A(p)$ depending on a vector of real parameters $p \in \mathbf{R}^{m}$ is the same as considered above except that the holomorphic dependence on parameters is changed to the smooth one. In this case, Theorem 2.4 is valid and allows finding derivatives $D^{h}$ of functions $C, C^{-1}, b_{i}^{k l}(t)$ of the order $|h| \leq s$, where $s$ is the degree of smoothness of the family $A(p)$. 
3. Transformation of families of real matrices to normal forms. Let us consider a family of real matrices $A: P \longrightarrow \mathbf{R}^{n^{2}}, P \subset \mathbf{R}^{m}$ smoothly depending on a vector of real parameters $p \in \mathbf{R}^{m}$ and determined in the vicinity of a point $p=p_{0}$. Let the matrix $A_{0}=A\left(p_{0}\right)$ have real eigenvalues $\lambda_{i}, i=1, \ldots, q$ and complex eigenvalues $\lambda_{i}, \overline{\lambda_{i}}, i=q+1, q+2, \ldots$ (overline denotes the complex conjugate operation). Then the Jordan form of the matrix $A_{0}$ is given by $J=\operatorname{Diag}\left(J_{1}, \ldots, J_{q}, J_{q+1}, \overline{J_{q+1}}, J_{q+2}\right.$, $\left.\overline{J_{q+2}}, \ldots\right)$, where the blocks $J_{i}$ correspond to the eigenvalues $\lambda_{i}$ and have the form $J_{i}=\operatorname{Diag}\left(J_{i}^{1}, J_{i}^{2}, \ldots\right) ; J_{i}^{k}$ are the Jordan blocks of dimensions $n_{k}\left(\lambda_{i}\right)$ beginning with the largest one.

Denote by $X_{i j A}^{k l}, X_{i j B}^{k l}, X_{i j C}^{k l}, X_{i j D}^{k l}$ blocks of an arbitrary matrix $X$ of the dimension $n \times n$ which are situated on the intersection of rows and columns belonging to the blocks $J_{i}^{k}$ and $J_{j}^{l}, J_{i}^{k}$ and $\overline{J_{j}^{l}}, \overline{J_{i}^{k}}$ and $J_{j}^{l}, \overline{J_{i}^{k}}$ and $\overline{J_{j}^{l}}$, respectively. Denote elements of these blocks by $x_{i j A}^{k l}(r, s), x_{i j B}^{k l}(r, s), x_{i j C}^{k l}(r, s)$, and $x_{i j D}^{k l}(r, s)$. In the case $i=j$ we will leave only one number in the subscript, e.g., $X_{i A}^{k l}, x_{i A}^{k l}(r, s)$.

Denote by $\operatorname{Rl} Y$ a decomplexification of a complex matrix $Y$ [3, pp. 244-245]

$$
\operatorname{Rl} Y=\left(\begin{array}{cc}
\operatorname{Re} Y & -\operatorname{Im} Y \\
\operatorname{Im} Y & \operatorname{Re} Y
\end{array}\right)
$$

Define a matrix $J_{R}=\operatorname{Diag}\left(J_{1}, \ldots, J_{q}, \mathrm{Rl} J_{q+1}, \mathrm{Rl} J_{q+2}, \ldots\right)$. The matrices $J_{R}$ and $J$ are connected by the relation $J_{R}=R J R^{-1}$, where $R=\operatorname{Diag}\left(I_{1}, \ldots, I_{q}, I_{q+1}^{\prime}, I_{q+2}^{\prime}, \ldots\right)$, $R^{-1}=\bar{R}=\operatorname{Diag}\left(I_{1}, \ldots, I_{q}, \overline{I_{q+1}^{\prime}}, \overline{I_{q+2}^{\prime}}, \ldots\right)$. The blocks $I_{j}^{\prime}$ have the form

$$
I_{j}^{\prime}=\frac{1+i}{2}\left(\begin{array}{cc}
I_{j} & -i I_{j} \\
-i I_{j} & I_{j}
\end{array}\right), \quad i=\sqrt{-1}
$$

where $I_{j}$ is the identity matrix of the same dimension as $J_{j}$. Let us choose the matrix $C_{0}$ transforming $A_{0}$ to the Jordan form $J(2.1)$ such that the matrices $C_{R 0}=C_{0} R^{-1}$ and $C_{R 0}^{-1}=R C_{0}^{-1}$ are real. For this purpose it is sufficient to choose the matrix $C_{0}$ so that its columns, passing through the blocks corresponding to real eigenvalues $J_{j}, j=1, \ldots, q$, are real, and columns, passing through the blocks corresponding to complex conjugate eigenvalues $J_{j}$ and $\overline{J_{j}}, j=q+1, q+2, \ldots$, are complex conjugate. This is possible due to the reality of $A_{0}$. Then each column of the matrix $C_{R 0}$ will be equal to the sum of the real and imaginary parts of the corresponding column of the matrix $C_{0}$. Using $C_{R 0}$ the matrix $A_{0}$ can be transformed to the form $J_{R}$

$$
A_{0}=C_{0} J C_{0}^{-1}=C_{R 0} J_{R} C_{R 0}^{-1} .
$$

It is shown in [8], [3, pp. 244-245] that the real miniversal deformation $A_{R}^{\prime}\left(p^{\prime}\right)$, $p^{\prime} \in \mathrm{R}^{d}$, to which an arbitrary family of real matrices $A(p), A\left(p_{0}\right)=A_{0}$ can be transformed by a smooth change of basis $C_{R}(p)$ and a smooth change of parameters $p^{\prime}=\varphi(p), \varphi\left(p_{0}\right)=\mathbf{0}$, can be chosen in the form $A_{R}^{\prime}\left(p^{\prime}\right)=J_{R}+B_{R}\left(p^{\prime}\right)$, $B_{R}=\operatorname{Diag}\left(B_{1}, \ldots, B_{q}, \mathrm{Rl} B_{q+1}, \mathrm{Rl} B_{q+2}, \ldots\right)$. Here $B_{j}$ is a block corresponding to the block $J_{j}$ whose elements are all zero, except at the places indicated in Figure $2.3(\mathrm{a}, \mathrm{b}$, or $\mathrm{c})$. In the blocks $B_{j}, j=1, \ldots, q$, the indicated places are filled by independent parameters $b_{j}^{k l}(t)_{a}$, and in the blocks $B_{j}, j=q+1, q+2, \ldots$, by complex numbers $b_{j}^{k l}(t)=b_{j}^{k l}(t)_{a}+i b_{j}^{k l}(t)_{b}$, where $b_{j}^{k l}(t)_{a}$ and $b_{j}^{k l}(t)_{b}$ are independent parameters. Numbering of parameters $b_{j}^{k l}(t)_{a}, b_{j}^{k l}(t)_{b}$ is carried out in the same way as in section 2. Let us introduce families of matrices $C=C_{R} R, C^{-1}=R^{-1} C_{R}^{-1}$, 
$A^{\prime}=J+B$, and $B=\operatorname{Diag}\left(B_{1}, \ldots, B_{q}, B_{q+1}, \overline{B_{q+1}}, B_{q+2}, \overline{B_{q+2}}, \ldots\right)$. Using expressions $A_{R}^{\prime}=J_{R}+B_{R}=R(J+B) R^{-1}=R A^{\prime} R^{-1}$, we obtain

$$
A(p)=C_{R}(p) A_{R}^{\prime}(\varphi(p)) C_{R}^{-1}(p)=C(p) A^{\prime}(\varphi(p)) C^{-1}(p) .
$$

The family $A^{\prime}\left(p^{\prime}\right)$ has the same structure as the complex miniversal deformation in section 2. This family as well as $A_{R}^{\prime}\left(p^{\prime}\right)$ is a miniversal deformation to which any family of real matrices $A(p), A\left(p_{0}\right)=A_{0}$ can be transformed in the vicinity of the point $p=p_{0}$, but it and the families $C, C^{-1}$ contain complex elements. For derivatives $D^{h}$ of the functions $p^{\prime}=\varphi(p), C(p)$, and $C^{-1}(p)$ transforming the family $A(p)$ to the form $A^{\prime}\left(p^{\prime}\right)$ the formulae of Theorem 2.4 are valid if we assume $b_{j}^{k l}(t)=b_{j}^{k l}(t)_{a}$ for $j \leq q$ and $b_{j}^{k l}(t)=b_{j}^{k l}(t)_{a}+i b_{j}^{k l}(t)_{b}$ for $j>q$. Using the relations $C_{R}=C R^{-1}$, $C_{R}^{-1}=R C^{-1}$ and Theorem 2.4, analogous formulae can be found for the derivatives $D^{h} C_{R}, D^{h} C_{R}^{-1}, D^{h} b_{j}^{k l}(t)_{a}$, and $D^{h} b_{j}^{k l}(t)_{b}$.

TheOREM 3.1. Let $A(p), p \in \mathbf{R}^{n}$ be a family of real matrices. Then the following formulae are valid for derivatives $D^{h},|h|>0$ of the functions $C_{R}(p), C_{R}^{-1}(p)$, $b_{j}^{k l}(t)_{a}(p)$, and $b_{j}^{k l}(t)_{b}(p)$ transforming the family $A(p)$ to the real miniversal deformation $A_{R}^{\prime}\left(p^{\prime}\right)$ described above (3.1)

$1^{\circ}$.

$$
\begin{gathered}
D^{h} b_{j}^{k l}(t)_{a}=\operatorname{Re} \frac{\operatorname{tr}^{(t)}\left[\left(R^{-1} F_{R} R\right)_{j A}^{k l}\right]}{\alpha_{j}^{k l}(t)}, \quad D^{h} b_{j}^{k l}(t)_{b}=\operatorname{Im} \frac{\operatorname{tr}^{(t)}\left[\left(R^{-1} F_{R} R\right)_{j A}^{k l}\right]}{\alpha_{j}^{k l}(t)}, \\
F_{R}=C_{R 0}^{-1}\left(D^{h} A-\sum_{\substack{h_{1}+h_{2}+h_{3}=h \\
h_{1}, h_{2}, h_{3} \neq h}} C_{h}^{h_{1} h_{2} h_{3}} D^{h_{1}} C_{R} D^{h_{2}} A_{R}^{\prime} D^{h_{3}} C_{R}^{-1}\right. \\
\left.+A_{0} \sum_{\substack{h_{1}+h_{2}=h \\
h_{1}, h_{2} \neq h}} C_{h}^{h_{1} h_{2}} D^{h_{1}} C_{R} D^{h_{2}} C_{R}^{-1}\right) C_{R 0} .
\end{gathered}
$$

$2^{\circ}$.

$$
\begin{aligned}
D^{h} C_{R} & =C_{R 0} R X R^{-1}, \\
G & =R^{-1}\left(D^{h} A_{R}^{\prime}-F_{R}\right) R .
\end{aligned}
$$

$3^{\circ}$

$$
D^{h} C_{R}^{-1}=-C_{R 0}^{-1} \sum_{\substack{h_{1}+h_{2}=h \\ h_{2} \neq h}} C_{h}^{h_{1} h_{2}} D^{h_{1}} C_{R} D^{h_{2}} C_{R}^{-1} .
$$

Here elements of the blocks $X_{i A}^{k l}$ and $X_{i D}^{k l}$ are expressed in terms of elements of the blocks $G_{i A}^{k l}$ and $G_{i D}^{k l}$, respectively, by formulae (2.8), and elements of other blocks of the matrix $X$ are expressed in terms of elements of the corresponding blocks of the matrix $G$ by formulae (2.7). In the last case it is necessary to take into account that the complex conjugate eigenvalues $\lambda_{i}, \overline{\lambda_{i}}$ are considered independently (for example, to obtain the formulae for elements of the block $X_{i j C}^{k l}$, it is necessary to replace in (2.7) $\left(\lambda_{i}-\lambda_{j}\right)$ by $\left(\overline{\lambda_{i}}-\lambda_{j}\right)$ and $g_{i j}^{k l}\left(r_{1}, s_{1}\right)$ by $\left.g_{i j C}^{k l}\left(r_{1}, s_{1}\right)\right)$.

Proof. The proof is based on the connection of the real and complex miniversal deformations (3.1). First let us consider a structure of the complex miniversal 
deformation $A^{\prime}\left(p^{\prime}\right)$ more thoroughly. As shown above, the matrix $A^{\prime}$ has a blockdiagonal form with blocks $A_{j A}^{\prime}, j \leq q$ and $A_{j A}^{\prime}, A_{j D}^{\prime}, j>q$ corresponding to the real and complex eigenvalues $\lambda_{j} \in \mathbf{R}, j \leq q$ and $\lambda_{j}, \overline{\lambda_{j}} \in \mathbf{C}, j>q$, respectively. The blocks $A_{j A}^{\prime}$ depend on parameters $b_{j}^{k l}(t)=b_{j}^{k l}(t)_{a}$ for $j \leq q$ and on parameters $b_{j}^{k l}(t)=b_{j}^{k l}(t)_{a}+i b_{j}^{k l}(t)_{b}$ for $j>q$. The blocks $A_{j D}^{\prime}=\overline{A_{j A}^{\prime}}, j>q$ depend on parameters $\overline{b_{j}^{k l}(t)}=b_{j}^{k l}(t)_{a}-i b_{j}^{k l}(t)_{b}$. Thus, we have a connection between the real and complex miniversal deformations, as well as transformation functions in the form

$$
\begin{gathered}
A^{\prime}=R^{-1} A_{R}^{\prime} R, \quad C=C_{R} R, \quad C^{-1}=R^{-1} C_{R}^{-1}, \\
b_{j}^{k l}(t)= \begin{cases}b_{j}^{k l}(t)_{a}, & j \leq q, \\
b_{j}^{k l}(t)_{a}+i b_{j}^{k l}(t)_{b}, & j>q .\end{cases}
\end{gathered}
$$

The formulae of theorem 2.4 are valid for derivatives of functions $b_{j}^{k l}(t)(p), C(p)$, and $C^{-1}(p)$. Combining these formulae with equalities (3.2) and (3.3), we can obtain expressions for derivatives of $b_{j}^{k l}(t)_{a}, b_{j}^{k l}(t)_{b}, C_{R}$, and $C_{R}^{-1}$.

Substituting relations (3.2) into formulae (2.5), (2.6), and (2.9), we obtain

$$
\begin{gathered}
F=R^{-1} F_{R} R, \\
D^{h} C_{R}=C_{R 0} R X R^{-1}, \\
G=R^{-1}\left(D^{h} A_{R}^{\prime}-F_{R}\right) R .
\end{gathered}
$$

It follows from expressions (3.3) and Theorem 2.4 that the derivatives $D^{h} b_{j}^{k l}(t)_{a}$ and $D^{h} b_{j}^{k l}(t)_{b}$ can be found by taking the real and imaginary parts of the right-hand side of relation (2.4) and using expression (3.4) for the matrix $F$. This proves item $1^{\circ}$ of the theorem.

Item $2^{\circ}$ follows directly from relations (3.5), (3.6), and Theorem 2.4. The fact that the matrix $D^{h} C_{R}$ calculated by these formulae will be real can be proved in the following way. Elements of the matrix $G=R^{-1} G_{R} R$, where $G_{R}=D^{h} A_{R}^{\prime}-F_{R}$ is a real matrix, have the properties $g_{i j A}^{k l}(r, s)=\overline{g_{i j D}^{k l}(r, s)}, g_{i j B}^{k l}(r, s)=\overline{g_{i j C}^{k l}(r, s)}$. It can be shown that elements of the matrix $X$ obtained from such a matrix $G$ have the same properties. It remains to note that then the matrix $R X R^{-1}$ and, consequently, the matrix $D^{h} C_{R}=C_{R 0} R X R^{-1}$ will be real.

For the proof of remaining item $3^{\circ}$ of the theorem it is sufficient to substitute the relations $C=C_{R} R$ and $C^{-1}=R^{-1} C_{R}^{-1}$ into expression (2.10).

For derivatives of the zero order $h=\mathbf{0}$ we have $D^{0} A_{R}^{\prime}=J_{R}, D^{0} C_{R}=C_{R 0}$, and $D^{0} C_{R}^{-1}=C_{R 0}^{-1}$. The recurrent procedure described in Theorem 3.1 allows finding derivatives $D^{h}$ of the real functions $C_{R}, C_{R}^{-1}, \varphi$, transforming the family of matrices $A(p)$ to the real miniversal deformation $A_{R}^{\prime}\left(p^{\prime}\right)$, for any $h$. Using these derivatives, the real family $A(p)$ can be transformed to the normal form with the accuracy up to $o\left(\left\|p-p_{0}\right\|^{s}\right)$, where $s$ is the degree of smoothness of the family.

3.1. Example. Let us consider a one-parameter family of real matrices $A(x)$ of the order $4 \times 4$ having at the point $x=0$ the pair of complex conjugate eigenvalues 
$\lambda=1+i, \bar{\lambda}=1-i$ with corresponding Jordan blocks of the order $n(\lambda)=2$ :

$$
A(x)=\left(\begin{array}{cccc}
1-4 x^{2} & 1+x & 2 x^{2} & 1 \\
-1+2 x & 1-x & 1-2 x^{2} & 3 x+2 x^{2} \\
2 x^{2} & 2 x^{2} & 1-2 x & -1+x \\
-x & 3 x & 1+4 x^{2} & 1+x
\end{array}\right) .
$$

The normal forms $A^{\prime}\left(p^{\prime}\right)$ and $A_{R}^{\prime}\left(p^{\prime}\right)$ of this family and the function $p^{\prime}=\varphi(x)$ determined using the recurrent procedure of Theorem 3.1 (up to $o\left(x^{2}\right)$ ) have the form

$$
\begin{gathered}
A_{R}^{\prime}\left(p^{\prime}\right)=\left(\begin{array}{cccc}
1 & 1 & -1 & 0 \\
b(2)_{a} & 1+b(1)_{a} & -b(2)_{b} & -1-b(1)_{b} \\
1 & 0 & 1 & 1 \\
b(2)_{b} & 1+b(1)_{b} & b(2)_{a} & 1+b(1)_{a}
\end{array}\right), \\
A^{\prime}\left(p^{\prime}\right)=\left(\begin{array}{cccc}
1+i & 1 & 0 & 0 \\
b(2) & 1+i+b(1) & 0 & 0 \\
0 & 0 & 1-i & 1 \\
0 & 0 & \overline{b(2)} & 1-i+\overline{b(1)}
\end{array}\right), \\
p^{\prime}=\left(b(1)_{a}, b(1)_{b}, b(2)_{a}, b(2)_{b}\right)^{T} \\
b(1)=b(1)_{a}+i b(1)_{b}=-x-2 x^{2}+i\left(-x-\frac{21}{8} x^{2}\right)+o\left(x^{2}\right), \\
b(2)=b(2)_{a}+i b(2)_{b}=-\frac{x}{2}+\frac{25}{8} x^{2}+i\left(\frac{3}{2} x-\frac{7}{4} x^{2}\right)+o\left(x^{2}\right) .
\end{gathered}
$$

4. Application to stability theory. As an application of miniversal deformations to the stability theory, let us consider a problem of investigation of the stability domain of a family of real $n \times n$ matrices $A(p), p \in \mathbf{R}^{m}$ in the vicinity of a singular boundary point. The stability domain of a family of matrices $A(p)$ is a set of values of the vector of parameters $p$ at which all eigenvalues $\lambda$ of the matrix $A(p)$ have negative real parts $\operatorname{Re} \lambda<0$. A boundary of the stability domain is characterized by matrices $A(p)$ having pure imaginary eigenvalues $\operatorname{Re} \lambda=0$, while for all other eigenvalues $\operatorname{Re} \lambda<0$. Nonsingular points of the stability boundary are characterized by the existence of only one simple zero eigenvalue $\lambda=0$ or one pair of simple complex conjugate eigenvalues $\lambda= \pm i \omega$ on the imaginary axis. Otherwise the boundary point is singular [2], [3, pp. 255-256]. A linear approximation of the stability domain at the boundary point is described by a tangent cone introduced by Levantovskii [10].

Definition 4.1. A tangent cone to the stability domain at the boundary point is a set of direction vectors $e=d p /\left.d \varepsilon\right|_{\varepsilon=0}$ of the curves $p(\varepsilon), \varepsilon>0$, which start at this point and lie in the stability domain.

A concept of the tangent cone can be illustrated by a simple example; see Figure 4.1. Here the tangent cone to the stability domain $S=\left\{p=\left(p_{1}, p_{2}\right)^{T}: 2 p_{2}>\right.$ $\left.p_{1}+p_{1}^{2}, 2 p_{1}+p_{1}^{2}>p_{2}\right\}$ at the origin has the form of an angle $K=\left\{e=\left(e_{1}, e_{2}\right)^{T}\right.$ : 


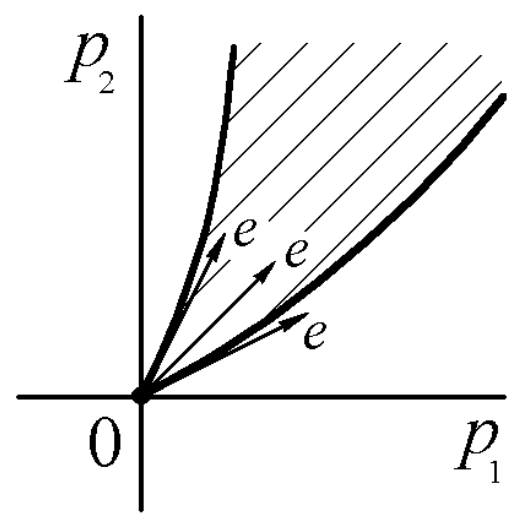

FIG. 4.1. A tangent cone to the stability domain.

$\left.2 e_{2} \geq e_{1}, 2 e_{1} \geq e_{2}\right\}$. Thus, the tangent cone consists of all vectors directed into the stability domain or tangent to its boundary.

In [10], using the miniversal deformation tangent cones have been described for some types of singular boundary points up to a linear diffeomorphism. In this section using the miniversal deformations and Theorem 2.4 expressions for tangent cones in the parameter space are found for all types of boundary points. It is shown that for determining the tangent cone we need only the Jordan form of the matrix $A$ and its first derivatives with respect to parameters at the boundary point under consideration.

Let $p=p_{0}$ be a boundary point of the stability domain and let $\lambda_{0}=0, \lambda_{j}=$ $i \omega_{j} \neq 0, \overline{\lambda_{j}}=-i \omega_{j}, j=1, \ldots, k$ be pure imaginary eigenvalues of the matrix $A\left(p_{0}\right)$ ( $i$ is the imaginary unit).

TheOrem 4.2. Assume that each pure imaginary eigenvalue $\lambda_{0}, \lambda_{j}, \overline{\lambda_{j}}, j=$ $1, \ldots, k$ of the matrix $A\left(p_{0}\right)$ has exactly one corresponding Jordan block of the dimension $n_{j}, j=0, \ldots, k$. If the system of vectors $f\left(l_{1}\right), g_{j}\left(l_{2}\right), h_{j}\left(l_{3}\right) \in \mathbf{R}^{m}, l_{1}=1, \ldots, n_{0}$, $l_{2}=1, \ldots, n_{j}, l_{3}=2, \ldots, n_{j}, j=1, \ldots, k$, is linearly independent, where

$$
\begin{aligned}
& f\left(l_{1}\right)=\left(\operatorname{tr}^{\left(l_{1}\right)}\left[F(1)_{0}^{11}\right], \ldots, \operatorname{tr}^{\left(l_{1}\right)}\left[F(m)_{0}^{11}\right]\right)^{T}, \\
& g_{j}\left(l_{2}\right)=\left(\operatorname{Retr}^{\left(l_{2}\right)}\left[F(1)_{j}^{11}\right], \ldots, \operatorname{Retr}^{\left(l_{2}\right)}\left[F(m)_{j}^{11}\right]\right)^{T}, \\
& h_{j}\left(l_{3}\right)=\left(\operatorname{Imtr}^{\left(l_{3}\right)}\left[F(1)_{j}^{11}\right], \ldots, \operatorname{Im~tr}^{\left(l_{3}\right)}\left[F(m)_{j}^{11}\right]\right)^{T}, \\
& F(r)=C_{0}^{-1} \frac{\partial A}{\partial p_{r}} C_{0},
\end{aligned}
$$

and derivatives are taken at $p=p_{0}$, then the tangent cone to the stability domain of the family $A(p)$ at the point $p=p_{0}$ has the form

$$
\begin{aligned}
& K=\left\{e \in \mathbf{R}^{m}:\right. \\
&(f(1), e) \leq 0,(f(2), e) \leq 0,(f(3), e)=\cdots=\left(f\left(n_{0}\right), e\right)=0, \\
&\left(g_{j}(1), e\right) \leq 0,\left(g_{j}(2), e\right) \leq 0,\left(g_{j}(3), e\right)=\cdots=\left(g_{j}\left(n_{j}\right), e\right)=0, \\
&\left.\left(h_{j}(2), e\right)=\cdots=\left(h_{j}\left(n_{j}\right), e\right)=0, j=1, \ldots, k\right\} .
\end{aligned}
$$

Here $(x, y)=x^{1} y^{1}+\cdots+x^{m} y^{m}$ is the scalar product in $\mathbf{R}^{m}$. In the case when zero 
is not an eigenvalue of the matrix $A\left(p_{0}\right)$ the expressions containing the vectors $f(l)$ have to be excluded from (4.2).

For the proof of the theorem we need the following lemmas.

LEMMA 4.3. The tangent cone to the stability domain of the family of polynomials $x^{n}-a_{1} x^{n-1}-\cdots-a_{n}$ at the point $a_{1}=\cdots=a_{n}=0$ in the parameter space $p=\left(a_{1}, \ldots, a_{n}\right)^{T} \in \mathbf{R}^{n}$ is equal to the set $\left\{a_{1} \leq 0, a_{2} \leq 0, a_{3}=\cdots=a_{n}=0\right\}$.

LEMMA 4.4. The tangent cone to the stability domain of the family of polynomials $z^{n}-\left(a_{1}+i b_{1}\right) z^{n-1}-\cdots-\left(a_{n}+i b_{n}\right)$ at the point $a_{1}=b_{1}=\cdots=a_{n}=b_{n}=0$ in the parameter space $p=\left(a_{1}, b_{1}, \ldots, a_{n}, b_{n}\right)^{T} \in \mathbf{R}^{2 n}$ is equal to the set $\left\{a_{1} \leq 0, b_{1} \in\right.$ $\left.\mathbf{R}, a_{2} \leq 0, b_{2}=a_{3}=\cdots=a_{n}=b_{n}=0\right\}$.

A polynomial is said to be stable if real parts of all its roots are negative. Lemmas 4.3 and 4.4 have been formulated and proved in [10].

Lemma 4.5. For any $\alpha_{1}^{j} \leq 0, \alpha_{2}^{j} \leq 0, j=0, \ldots, k$, there exists a curve $q_{1}(\varepsilon), q_{1}(0)=\mathbf{0}$ in the space $q_{1}=\left(a_{l_{1}}^{j_{1}}, b_{l_{2}}^{j_{2}}, l_{2} \neq 1\right)^{T}$ of all coefficients (except $\left.b_{1}^{1}, \ldots, b_{1}^{k}\right)$ of the polynomials

$$
\begin{gathered}
x^{n_{0}}-a_{1}^{0} x^{n_{0}-1}-\cdots-a_{n_{0}}^{0}, \\
z^{n_{j}}-\left(a_{1}^{j}+i b_{1}^{j}\right) z^{n_{j}-1}-\cdots-\left(a_{n_{j}}^{j}+i b_{n_{j}}^{j}\right), \quad j=1, \ldots, k,
\end{gathered}
$$

such that all polynomials (4.3) are stable along the curve at $\varepsilon>0$, the vector of coefficients $q_{2}=\left(b_{1}^{1}, \ldots, b_{1}^{k}\right)^{T}$ is related with $q_{1}$ and $\varepsilon$ by an arbitrary given smooth function $q_{2}=Q_{2}\left(q_{1}, \varepsilon\right), Q_{2}(\mathbf{0}, 0)=\mathbf{0}$, and the following conditions are fulfilled:

$$
\begin{gathered}
\frac{d a_{1}^{j}}{d \varepsilon}=\alpha_{1}^{j}, \frac{d a_{2}^{j}}{d \varepsilon}=\alpha_{2}^{j}, \frac{d a_{3}^{j}}{d \varepsilon}=\cdots=\frac{d a_{n_{j}}^{j}}{d \varepsilon}=0, j=0, \ldots, k, \\
\frac{d b_{2}^{j}}{d \varepsilon}=\cdots=\frac{d b_{n_{j}}^{j}}{d \varepsilon}=0, j=1, \ldots, k .
\end{gathered}
$$

Proof. Let us consider polynomials of the form

$$
\begin{gathered}
\left(x+\varepsilon^{2}\right)^{n_{0}-2}\left(x^{2}+\left(-\alpha_{1}^{0} \varepsilon+\varepsilon^{2}\right) x-\alpha_{2}^{0} \varepsilon+\varepsilon^{2}\right), \\
\left(z+\varepsilon^{2}\right)^{n_{j}-2}\left(z^{2}+\left(-\left(\alpha_{1}^{j}+i b_{1}^{j}\right) \varepsilon+\varepsilon^{2}\right) z-\alpha_{2}^{j} \varepsilon+\varepsilon^{2}\right) .
\end{gathered}
$$

These polynomials are stable at $\varepsilon>0$ and their coefficients, obtained after performing multiplication, satisfy conditions (4.4). Expressing these coefficients through $q_{2}=$ $\left(b_{1}^{1} \varepsilon, \ldots, b_{1}^{k} \varepsilon\right)^{T}, \varepsilon$ and then substituting the obtained expressions into the relation $q_{2}=Q_{2}\left(q_{1}, \varepsilon\right)$, we obtain $q_{2}=Q_{2}^{\prime}\left(q_{2}, \varepsilon\right)$. Moreover, the matrix of the derivatives $\partial Q_{2}^{\prime} / \partial q_{2}$ is the zero matrix at $\varepsilon=0$. Hence, by the implicit function theorem the vector $q_{2}$ is a smooth function $q_{2}=Q_{2}^{\prime \prime}(\varepsilon)$ in the vicinity of $\varepsilon=0$. If we substitute the function $q_{2}=\left(b_{1}^{j}\right)^{T}=Q_{2}^{\prime \prime}(\varepsilon)$ into (4.5), the coefficients of polynomials (4.5) will form the desired curve $q_{1}=q_{1}(\varepsilon)$.

Proof of Theorem 4.2. Using expression (2.2) the characteristic equation of the matrix $A(p)$ in the vicinity of the point $p_{0}$ can be written in the form

$$
\operatorname{det}(A(p)-\lambda E)=\operatorname{det}\left(C(p) A^{\prime}(\varphi(p)) C^{-1}(p)-\lambda E\right)=\operatorname{det}\left(A^{\prime}(\varphi(p))-\lambda E\right)=0,
$$

where $A^{\prime}\left(p^{\prime}\right)$ is the miniversal deformation of the matrix $A\left(p_{0}\right)$. Hence, stability of the matrix $A(p)$ is equivalent to stability of the matrix $A^{\prime}(\varphi(p))$ (normal form) and, by virtue of the block-diagonal structure of $A^{\prime}$, it is equivalent to simultaneous stability of 
all its blocks. The blocks corresponding to eigenvalues of the matrix $A_{0}$ with negative real parts are stable at the point $p_{0}$ and, consequently, in the vicinity of this point. Thus, stability in the vicinity of $p_{0}$ is characterized by stability of the blocks $A_{0}^{\prime}, A_{j}^{\prime}$, $\overline{A_{j}^{\prime}}, j=1, \ldots, k$, corresponding to pure imaginary eigenvalues or, since the blocks $A_{j}^{\prime}$ and $\overline{A_{j}^{\prime}}$ are stable or unstable simultaneously (because of complex conjugacy; see section 3 ), by stability of the blocks $A_{0}^{\prime}, A_{j}^{\prime}, j=1, \ldots, k$. Choosing the blocks $A_{0}^{\prime}, A_{j}^{\prime}$ as the blocks of the type (a) (see Figure 2.2), and taking into account that there is only one Jordan block corresponding to each eigenvalue, their characteristic equations take the form

$$
\begin{aligned}
\operatorname{det}\left(A_{0}^{\prime}-\lambda E\right)= & \lambda^{n_{0}}-b_{0}^{11}(1)_{a} \lambda^{n_{0}-1}-\cdots-b_{0}^{11}\left(n_{0}\right)_{a}=0, \\
\operatorname{det}\left(A_{j}^{\prime}-\lambda E\right)= & \eta^{n_{j}}-\left(b_{j}^{11}(1)_{a}+i b_{j}^{11}(1)_{b}\right) \eta^{n_{j}-1}-\cdots \\
& \cdots-\left(b_{j}^{11}\left(n_{j}\right)_{a}+i b_{j}^{11}\left(n_{j}\right)_{b}\right)=0, \\
& \eta=\lambda-i \omega_{j}, \quad j=1, \ldots, k,
\end{aligned}
$$

where $b_{0}^{11}\left(l_{1}\right)_{a}, b_{j}^{11}\left(l_{2}\right)_{a}$, and $b_{j}^{11}\left(l_{3}\right)_{b}$ are parameters of the miniversal deformation smoothly depending on $p$. By Theorem 2.4, and using expressions (4.1), we get

$$
\begin{gathered}
\nabla b_{0}^{11}\left(l_{1}\right)_{a}=f\left(l_{1}\right), \quad \nabla b_{j}^{11}\left(l_{2}\right)_{a}=g_{j}\left(l_{2}\right), \quad \nabla b_{j}^{11}\left(l_{3}\right)_{b}=h_{j}\left(l_{3}\right), \\
\nabla=\left(\frac{\partial}{\partial p_{1}}, \ldots, \frac{\partial}{\partial p_{m}}\right)^{T} .
\end{gathered}
$$

Let us consider an arbitrary smooth curve $p=p(\varepsilon)$ with a direction $e=d p / d \varepsilon$. Coefficients of polynomials (4.6) along this curve have the form

$$
\begin{aligned}
& b_{0}^{11}\left(l_{1}\right)_{a}(\varepsilon)=\left(f\left(l_{1}\right), e\right) \varepsilon+O\left(\varepsilon^{2}\right), \\
& b_{j}^{11}\left(l_{2}\right)_{a}(\varepsilon)=\left(g_{j}\left(l_{2}\right), e\right) \varepsilon+O\left(\varepsilon^{2}\right), \\
& b_{j}^{11}\left(l_{2}\right)_{b}(\varepsilon)=\left(h_{j}\left(l_{2}\right), e\right) \varepsilon+O\left(\varepsilon^{2}\right) .
\end{aligned}
$$

Applying Lemmas 4.3 and 4.4 to polynomials (4.6) and taking into account expressions (4.8), we obtain that conditions (4.2) are necessary for the curve $p(\varepsilon)$ to lie in the stability domain.

Let us prove that conditions (4.2) are also sufficient. Denote by $q$ a vector whose components are $b_{0}^{11}\left(l_{1}\right)_{a}, b_{j}^{11}\left(l_{2}\right)_{a}$, and $b_{j}^{11}\left(l_{3}\right)_{b}$, and by $q_{1}, q_{2}$ the parts of $q$ so that $q_{2}=\left(b_{1}^{11}(1)_{b}, \ldots, b_{k}^{11}(1)_{b}\right)^{T}$ and $q_{1}$ consists of $d^{\prime}=n_{0}+2 n_{1}+\cdots+2 n_{k}-k$ remaining components of the vector $q$. The vectors $q, q_{1}$, and $q_{2}$ are connected with $p$ by smooth functions $q=Q(p), q_{1}=Q_{1}(p)$, and $q_{2}=Q_{2}(p)$, which are parts of the mapping $p^{\prime}=\varphi(p)$. The Jacobian $d Q_{1} / d p$ is a $d^{\prime} \times m$ matrix whose rows $f\left(l_{1}\right), g_{j}\left(l_{2}\right), h_{j}\left(l_{3}\right)$, $l_{3} \neq 1$ form, according to the conditions of the theorem, a linear independent system. Let $p_{1}$ be the vector consisting of $d^{\prime}$ components of the vector $p$, which corresponds to a linearly independent system of columns of this Jacobian, and denote by $p_{2}$ the vector consisting of the remaining components of $p$. Then $\operatorname{det}\left[d Q_{1} / d p_{1}\right] \neq 0$ at the point $p=p_{0}$, and by the implicit function theorem $p_{1}=P_{1}\left(q_{1}, p_{2}\right)$ in the vicinity of the point $p=p_{0}, q_{1}=\mathbf{0}$. By substituting this relation into $q_{2}=Q_{2}\left(p_{1}, p_{2}\right)$, we get $q_{2}=Q_{2}^{\prime}\left(q_{1}, p_{2}\right)$. Let us consider an arbitrary direction $e$ satisfying conditions (4.2) and assume that $p_{2}=e_{2} \varepsilon$, where $e_{1}$ and $e_{2}$ are the parts of the vector $e$ corresponding to $p_{1}$ and $p_{2}$, respectively. Then $q_{2}=Q_{2}^{\prime}\left(q_{1}, e_{2} \varepsilon\right)=Q_{2}^{\prime \prime}\left(q_{1}, \varepsilon\right)$. By Lemma 4.5 there exists 
a curve $q_{1}=q_{1}(\varepsilon)$ such that $d q_{1} / d \varepsilon=\left(d Q_{1} / d p\right) e, q_{2}=Q_{2}^{\prime \prime}\left(q_{1}, \varepsilon\right)$ and all polynomials (4.6) are stable at $\varepsilon>0$ (conditions (4.4) of the lemma are fulfilled due to (4.2), (4.8)). Then the curve $p=p(\varepsilon), \varepsilon>0$ such that $p_{1}=P_{1}\left(q_{1}(\varepsilon), e_{2} \varepsilon\right), p_{2}=e_{2} \varepsilon$ lies in the stability domain of the family $A(p)$ and has the direction $e$, which was chosen as an arbitrary direction from the set (4.2).

Theorem 4.2 covers a case when all pure imaginary eigenvalues of $A\left(p_{0}\right)$ are nonderogatory (have only one corresponding Jordan block). In the general case, when some pure imaginary eigenvalues of $A\left(p_{0}\right)$ have several corresponding Jordan blocks, the tangent cone to the stability domain is no longer a product of lines and half-lines as in (4.2). Investigation of this case is much more complicated. However, using an approach presented in the proof of Theorem 4.2, it is possible to find sets of directions belonging to the tangent cone for a boundary point of an arbitrary type.

THEOREM 4.6. Let $\lambda_{0}=0, \lambda_{j}, \overline{\lambda_{j}}, j=1, \ldots, k$ be pure imaginary eigenvalues of the matrix $A\left(p_{0}\right)$ and let each eigenvalue $\lambda_{j}, j=0, \ldots, k$ have corresponding Jordan blocks of the dimensions $n_{1}\left(\lambda_{j}\right) \geq n_{2}\left(\lambda_{j}\right) \geq \cdots$. Assume that the system of vectors $f^{r s}\left(l_{1}\right), g_{j}^{r s}\left(l_{2}\right), h_{j}^{r s}\left(l_{3}\right) \in \mathbf{R}^{m}$ for $r \geq s$ and $l_{3} \neq 1$ at $r=s$ is linearly independent, where

$$
\begin{aligned}
f^{r s}\left(l_{1}\right)= & \left(\operatorname{tr}^{\left(l_{1}\right)}\left[F(1)_{0}^{r s}\right], \ldots, \operatorname{tr}^{\left(l_{1}\right)}\left[F(m)_{0}^{r s}\right]\right)^{T}, \\
g_{j}^{r s}\left(l_{2}\right)= & \left(\operatorname{Retr}^{\left(l_{2}\right)}\left[F(1)_{j}^{r s}\right], \ldots, \operatorname{Retr}^{\left(l_{2}\right)}\left[F(m)_{j}^{r s}\right]\right)^{T}, \\
h_{j}^{r s}\left(l_{3}\right)= & \left(\operatorname{Imtr}^{\left(l_{3}\right)}\left[F(1)_{j}^{r s}\right], \ldots, \operatorname{Im}_{\left.\operatorname{tr}^{\left(l_{3}\right)}\left[F(m)_{j}^{r s}\right]\right)^{T},}\right. \\
& F(r)=C_{0}^{-1} \frac{\partial A}{\partial p_{r}} C_{0},
\end{aligned}
$$

and the derivatives are taken at $p=p_{0}$. Then the set

$$
K_{1}=\left\{e \in \mathbf{R}^{m}:\right.
$$

$$
\begin{aligned}
& \left(f^{r r}(1), e\right) \leq 0,\left(f^{r r}(2), e\right) \leq 0,\left(f^{r r}(3), e\right)=\cdots=\left(f^{r r}\left(n_{r}\left(\lambda_{0}\right)\right), e\right)=0, \\
& \left(g_{j}^{r r}(1), e\right) \leq 0,\left(g_{j}^{r r}(2), e\right) \leq 0,\left(g_{j}^{r r}(3), e\right)=\cdots=\left(g_{j}^{r r}\left(n_{r}\left(\lambda_{j}\right)\right), e\right)=0, \\
& \left(h_{j}^{r r}(2), e\right)=\cdots=\left(h_{j}^{r r}\left(n_{r}\left(\lambda_{j}\right)\right), e\right)=0, \\
& \left.\left(f^{r s}\left(l_{1}\right), e\right)=\left(g_{j}^{r s}\left(l_{2}\right), e\right)=\left(h_{j}^{r s}\left(l_{2}\right), e\right)=0, r>s, j=1, \ldots, k\right\}
\end{aligned}
$$

belongs to the tangent cone to the stability domain at the point $p_{0}$. Analogously, if the system of vectors (4.9) for $r \leq s$ and $l_{3} \neq 1$ at $r=s$ is linearly independent, then the set

$$
\begin{aligned}
K_{2}=\{ & e \in \mathbf{R}^{m}: \\
& \left(f^{r r}(1), e\right) \leq 0,\left(f^{r r}(2), e\right) \leq 0,\left(f^{r r}(3), e\right)=\cdots=\left(f^{r r}\left(n_{r}\left(\lambda_{0}\right)\right), e\right)=0, \\
& \left(g_{j}^{r r}(1), e\right) \leq 0,\left(g_{j}^{r r}(2), e\right) \leq 0,\left(g_{j}^{r r}(3), e\right)=\cdots=\left(g_{j}^{r r}\left(n_{r}\left(\lambda_{j}\right)\right), e\right)=0, \\
& \left(h_{j}^{r r}(2), e\right)=\cdots=\left(h_{j}^{r r}\left(n_{r}\left(\lambda_{j}\right)\right), e\right)=0, \\
& \left.\left.\left(f^{r s}\left(l_{1}\right), e\right)=\left(g_{j}^{r s}\left(l_{2}\right), e\right)=h_{j}^{r s}\left(l_{2}\right), e\right)=0, r<s, j=1, \ldots, k,\right\}
\end{aligned}
$$

belongs to the tangent cone. 
Proof. Characteristic equations of the blocks $A_{j}^{\prime}$ in this case cannot be written explicitly as in (4.6). However, if we consider curves $p=p(\varepsilon)$ lying on the surface determined by the equations

$$
b_{0}^{r s}\left(l_{1}\right)_{a}(p)=b_{j}^{r s}\left(l_{2}\right)_{a}(p)=b_{j}^{r s}\left(l_{2}\right)_{b}(p)=0, \quad r>s, \quad j=1, \ldots, k,
$$

the blocks $A_{j}^{\prime}$ will have block-triangular form, and their characteristic equations along these curves will take the form

$$
\begin{array}{r}
\operatorname{det}\left(A_{0}^{\prime}-\lambda E\right)=\prod_{r}\left(\lambda^{n_{r}\left(\lambda_{0}\right)}-b_{0}^{r r}(1)_{a} \lambda^{n_{r}\left(\lambda_{0}\right)-1}-\cdots-b_{0}^{r r}\left(n_{r}\left(\lambda_{0}\right)\right)_{a}\right)=0 \\
\operatorname{det}\left(A_{j}^{\prime}-\lambda E\right)=\prod_{r}\left(\eta^{n_{r}\left(\lambda_{j}\right)}-\left(b_{j}^{r r}(1)_{a}+i b_{j}^{r r}(1)_{b}\right) \eta^{n_{r}\left(\lambda_{j}\right)-1}-\cdots\right. \\
\left.\cdots-\left(b_{j}^{r r}\left(n_{r}\left(\lambda_{j}\right)\right)_{a}+i b_{j}^{r r}\left(n_{r}\left(\lambda_{j}\right)\right)_{b}\right)\right)=0, \quad \eta=\lambda-i \omega_{j}, \quad j=1, \ldots, k .
\end{array}
$$

By Theorem 2.4 and using expressions (4.9), we obtain

$$
\nabla b_{0}^{r s}\left(l_{1}\right)_{a}=f^{r s}\left(l_{1}\right), \quad \nabla b_{j}^{r s}\left(l_{2}\right)_{a}=g_{j}^{r s}\left(l_{2}\right), \quad \nabla b_{j}^{r s}\left(l_{3}\right)_{b}=h_{j}^{r s}\left(l_{3}\right) .
$$

Since the system of the vectors $f^{r s}\left(l_{1}\right), g_{j}^{r s}\left(l_{2}\right), h_{j}^{r s}\left(l_{2}\right), r>s$ is linearly independent, by the implicit function theorem (4.12) can be solved with respect to a part of variables $p_{1}=P_{1}\left(p_{2}\right)$, where $p_{1}$ and $p_{2}$ are parts of the vector $p$. Then the coefficients of polynomials (4.13) depend only on the vector of parameters $p_{2}$, and the direction of any curve $p_{1}=P_{1}\left(p_{2}(\varepsilon)\right), p_{2}=p_{2}(\varepsilon)$ satisfies all the conditions standing in the last row of (4.10). For the proof of the theorem it is sufficient to find a curve $p_{2}=p_{2}(\varepsilon)$ having an arbitrary chosen direction $e_{2}$ such that the vector $e$ consisting of $e_{1}=\left(d P_{1} / d p_{2}\right) e_{2}$ and $e_{2}$ satisfies all conditions (4.10), and all polynomials (4.13) are stable along this curve (conditions in the last row of (4.10) are satisfied automatically due to the choice of $e_{1}$ ). This problem coincides with the problem solved in the proof of Theorem 4.2.

To prove that the set (4.11) belongs to the tangent cone, one should consider curves lying in the surface

$$
b_{0}^{r s}\left(l_{1}\right)_{a}(p)=b_{j}^{r s}\left(l_{2}\right)_{a}(p)=b_{j}^{r s}\left(l_{2}\right)_{b}(p)=0, \quad r<s, \quad j=1, \ldots, k .
$$

Then the proof is carried out analogously.

The relations determining sets (4.10) and (4.11) are sufficient conditions that a direction belongs to the tangent cone. Note that Burke and Overton [4] obtained necessary conditions for the stable perturbations of the matrix in the general case, which can be used as necessary conditions that a direction belongs to the tangent cone.

In the nonderogatory case, sets (4.10) and (4.11) coincide and are equal to the tangent cone by Theorem 4.2. Theorems 4.2 and 4.6 give a constructive method for determining the tangent cone to the stability domain at the boundary point. For this purpose we need only information about the matrix $A$ and its first derivatives with respect to parameters at the point under consideration.

In the case of Theorem 4.2 for determining the tangent cone, we need to transform the matrix $A_{0}=A\left(p_{0}\right)$ to the Jordan form (2.1) and find the derivatives $\partial A / \partial p_{r}$ at the point under consideration. Note that components of vectors (4.1) are sums of products of some row of the matrix $C_{0}^{-1}$ by the matrix $\partial A / \partial p_{r}$ and by some column of the matrix $C_{0}$. Moreover, only the columns and rows of the matrices $C_{0}$ and $C_{0}^{-1}$, 
corresponding to the blocks of pure imaginary eigenvalues, are used. This fact can be used to reduce the amount of calculations for determining the tangent cone.

It is well known that the columns of the matrix $C_{0}$ corresponding to the block $A_{j}^{\prime}$ (in the nonderogatory case) are the eigenvector and associated vectors $u_{j}^{0}, \ldots, u_{j}^{n_{j}-1}$ (also called generalized eigenvectors) of the eigenvalue $\lambda_{j}$

$$
\begin{aligned}
A_{0} u_{j}^{0}= & \lambda_{j} u_{j}^{0}, \\
A_{0} u_{j}^{1}= & \lambda_{j} u_{j}^{1}+u_{j}^{0}, \\
& \vdots \\
A_{0} u_{j}^{n_{j}-1}= & \lambda_{j} u_{j}^{n_{j}-1}+u_{i}^{n_{j}-2} .
\end{aligned}
$$

Then the rows of the matrix $C_{0}^{-1}$ corresponding to the same block are determined as the left eigenvector and associate vectors $v_{j}^{n_{j}-1}, \ldots, v_{j}^{0}$, taken in reverse order,

$$
\begin{gathered}
v_{j}^{0} A_{0}=\lambda_{j} v_{j}^{0}, \\
v_{j}^{1} A_{0}=\lambda_{j} v_{j}^{1}+v_{j}^{0}, \\
\vdots \\
v_{j}^{n_{j}-1} A_{0}=\lambda_{j} v_{j}^{n_{j}-1}+v_{i}^{n_{j}-2},
\end{gathered}
$$

and satisfying the normalization conditions

$$
v_{j}^{0} u_{j}^{n_{j}-1}=1, \quad v_{j}^{l} u_{j}^{n_{j}-1}=0, \quad l=1, \ldots, n_{j}-1 .
$$

Using these properties of the matrices $C_{0}$ and $C_{0}^{-1}$ expressions (4.1) can be written in the form

$$
\begin{aligned}
f\left(l_{1}\right) & =\left(\sum_{t=0}^{n_{0}-l_{1}} v_{0}^{t} \frac{\partial A}{\partial p_{1}} u_{0}^{n_{0}-l_{1}-t}, \ldots, \sum_{t=0}^{n_{0}-l_{1}} v_{0}^{t} \frac{\partial A}{\partial p_{m}} u_{0}^{n_{0}-l_{1}-t}\right)^{T}, \\
g_{j}\left(l_{2}\right) & =\left(\operatorname{Re} \sum_{t=0}^{n_{j}-l_{2}} v_{j}^{t} \frac{\partial A}{\partial p_{1}} u_{j}^{n_{j}-l_{2}-t}, \ldots, \operatorname{Re} \sum_{t=0}^{n_{j}-l_{2}} v_{j}^{t} \frac{\partial A}{\partial p_{m}} u_{j}^{n_{j}-l_{2}-t}\right)^{T}, \\
h_{j}\left(l_{3}\right) & =\left(\operatorname{Im} \sum_{t=0}^{n_{j}-l_{3}} v_{j}^{t} \frac{\partial A}{\partial p_{1}} u_{j}^{n_{j}-l_{3}-t}, \ldots, \operatorname{Im} \sum_{t=0}^{n_{j}-l_{3}} v_{j}^{t} \frac{\partial A}{\partial p_{m}} u_{j}^{n_{j}-l_{3}-t}\right)^{T} .
\end{aligned}
$$

According to (4.17), for determining the vectors $f\left(l_{1}\right), g_{j}\left(l_{2}\right)$, and $h_{j}\left(l_{3}\right)$ we need to know only right and left eigenvectors and associated vectors corresponding to pure imaginary eigenvalues and satisfying normalization conditions (4.16), and the derivatives $\partial A / \partial p_{r}, r=1, \ldots, m$ at the point $p=p_{0}$.

Analogous expressions can be obtained for vectors (4.9). For this purpose in expressions (4.17) one should substitute $f^{r s}\left(l_{1}\right), g_{j}^{r s}\left(l_{2}\right), h_{j}^{r s}\left(l_{3}\right), u_{j s}^{t}, v_{j r}^{t}, n_{0}^{\prime}, n_{j}^{\prime}$ instead of $f\left(l_{1}\right), g_{j}\left(l_{2}\right), h_{j}\left(l_{3}\right), u_{j}^{t}, v_{j}^{t}, n_{0}, n_{j}$, respectively, where $n_{0}^{\prime}=\min \left(n_{r}\left(\lambda_{0}\right), n_{s}\left(\lambda_{0}\right)\right)$, $n_{j}^{\prime}=\min \left(n_{r}\left(\lambda_{j}\right), n_{s}\left(\lambda_{j}\right)\right)$, and $u_{j r}^{t} v_{j r}^{t}, t=0, \ldots, n_{r}\left(\lambda_{j}\right)-1$ are right and left eigenvectors and associated vectors corresponding to the Jordan block $J_{j}^{r}$ of the eigenvalue $\lambda_{j}$. In this case the normalization conditions for the vectors $u_{j r}^{t_{1}}, v_{j r}^{t_{2}}$ take the form (4.16), while the normalization conditions for the vectors $u_{j r}^{t_{1}}, v_{j s}^{t_{2}}, r \neq s$ are

$$
v_{j r}^{n_{r}\left(\lambda_{j}\right)-1} u_{j s}^{n_{s}\left(\lambda_{j}\right)-t}=0, \quad t=1, \ldots, n_{j}^{\prime}, \quad j=0, \ldots, k .
$$


Note that Theorems 4.2 and 4.6 are valid in the case of families of complex matrices smoothly depending on a vector of real parameters, if the vectors $g_{j}\left(l_{1}\right), h_{j}\left(l_{2}\right)$, and $g_{j}^{r s}\left(l_{1}\right), h_{j}^{r s}\left(l_{2}\right)$ are determined separately for each pure imaginary eigenvalue of the matrix $A_{0}$ (also for the zero eigenvalue and for each of the complex conjugate eigenvalues, if any) and the expressions containing the vectors $f(l)$ and $f^{r s}(l)$ are excluded from (4.2), (4.10), and (4.11).

If we consider generic families of matrices $[1,2,3]$ the condition of linearly independence of the system of vectors in Theorem 4.2 is satisfied. It follows from the fact that, in the generic case, the codimension of a surface in the parameter space $\mathbf{R}^{m}$ (stratum), on which all the matrices $A(p)$ have the same type of Jordan structure of pure imaginary eigenvalues of the matrix $A_{0}$, is equal to $d^{\prime}=n_{0}+2 n_{1}+\cdots+2 n_{k}-k$ (the number of parameters of the miniversal deformation, which correspond to the blocks of pure imaginary eigenvalues minus the number of nonzero pure imaginary eigenvalues, whose magnitudes are arbitrary on the stratum). There is a field of vectors (4.1) determined on this surface ( $d^{\prime}$ vectors at each point). The condition that the system of these vectors is linearly dependent leads to $m$ equations at each point of the surface, where components of one vector are expressed as a linear combination of corresponding components of the other vectors with $\left(d^{\prime}-1\right)$ arbitrary coefficients. These equations are equivalent to $\left(m-d^{\prime}+1\right)$ equations which do not contain these coefficients. But, since the dimension of the stratum is equal to $m-d^{\prime}$ (less than the number of equations), in the generic case these equations have no solution on the stratum. This means that the system of vectors is linearly independent. The analogous statement is correct in the case of Theorem 4.6. Thus, Theorems 4.2 and 4.6 can be applied to all points of a boundary of the stability domain of a generic family of matrices.

5. Conclusion. In this paper the problem of transformation of families of matrices to the Arnold's and Galin's normal forms (miniversal deformations) is solved. A constructive method determining a change of basis and a change of parameters, transforming a family of real or complex matrices to the normal form in the vicinity of the initial value of the parameter vector, is suggested. Finding the miniversal deformation of a family of matrices and describing the transformation to it represent a complete procedure of transformation to the normal form (2.2). The first step in this procedure is transformation of the matrix $A_{0}=A\left(p_{0}\right)$ to the Jordan form (2.1) and finding the miniversal deformation $A^{\prime}\left(p^{\prime}\right)$ according to $[1,2,3]$. Then, using Theorems 2.4 or 3.1, the derivatives of the families $C(p), C^{-1}(p)$ and of the mapping $p^{\prime}=\varphi(p)$, with respect to the parameters at the point $p=p_{0}$, are determined. At this step a recurrent procedure is used. Note that the procedure consists of only explicit relations containing elementary arithmetic operations. Using the obtained derivatives, the desired functions $p^{\prime}=\varphi(p)$ and $C(p), C^{-1}(p)$ (describing a change of parameters and a change of basis) are found as Taylor series. Thus, the procedure of transformation of a matrix family to the normal form is complete.

The spectrum of the normal form $A^{\prime}(\varphi(p))$ coincides with the spectrum of the family $A(p)$, but $A^{\prime}\left(p^{\prime}\right)$ is a family of sparse matrices depending on parameters in a quite simple way. This makes it efficient to apply normal forms (if the mapping $p^{\prime}=\varphi(p)$ is known) to investigation of different properties of the spectrum of the family $A(p)$. In section 4 the stability domain of a family of matrices in the vicinity of the singular boundary point $p=p_{0}$ is studied. In the cases when all pure imaginary eigenvalues of the matrix $A\left(p_{0}\right)$ are nonderogatory, tangent cones to the stability domain at the point $p=p_{0}$ are found. In the case of an arbitrary Jordan structure 
of $A\left(p_{0}\right)$, sufficient conditions, which describe directions belong to the tangent cone, are found. These conditions describe subsets of the tangent cones. It turns out that tangent cones are determined by the right and left eigenvectors and associated vectors corresponding to pure imaginary eigenvalues and the first derivatives of the matrix $A(p)$ with respect to parameters at the point $p=p_{0}$. Expressions determining the tangent cone are explicit and simple. They can be used in different stability problems studying dependence on parameters.

Moreover, by means of using transformation to the normal form, it is possible to investigate local properties of the spectral radius, to solve the problems of finding eigenvalues of perturbed matrices, etc. In this connection it is convenient to investigate the collapse of nonderogatory multiple eigenvalues. In this case the perturbed eigenvalues are determined as roots of a polynomial whose coefficients are obtained from Theorem 2.4.

Acknowledgments. The author thanks Alexander P. Seyranian for his attention to this work and helpful discussions, and Wolfhard Kliem and Michael L. Overton for useful comments.

\section{REFERENCES}

[1] V. I. Arnold, On matrices depending on parameters, Russian Math. Surveys, 26 (1971), pp. $29-43$.

[2] V. I. ARnold, Lectures on bifurcations and versal families, Russian Math. Surveys, 27 (1972), pp. 119-184.

[3] V. I. ARnold, Geometrical Methods in the Theory of Ordinary Differential Equations, Springer-Verlag, New York, 1983.

[4] J. V. Burke And M. L. Overton, Stable perturbations of nonsymmetric matrices, Linear Algebra Appl., 171 (1992), pp. 249-273.

[5] R. Cushman, A. Kelley, and H. KoçaK, Versal normal form at the Lagrangian equilibrium $L_{4}$, J. Differential Equations, 64 (1986), pp. 340-374.

[6] A. Edelman, E. Elmroth, And B. Kågström, A geometric approach to perturbation theory of matrices and matrix pencils. I. Versal deformations, SIAM J. Matrix Anal. Appl., 18 (1997), pp. 653-692.

[7] A. Edelman and H. Murakami, Polynomial roots from companion matrix eigenvalues, Math. Comp., 64 (1995), pp. 763-776.

[8] D. M. Galin, On real matrices depending on parameters, Uspekhi Mat. Nauk, 27 (1972), pp. 241-242 (in Russian).

[9] F. R. Gantmacher, The Theory of Matrices, Vols. 1, 2, Chelsea, New York, 1960.

[10] L. V. LevantovskiI, The boundary of a set of stable matrices, Russian Math. Surveys, 35 (1980), pp. 249-250.

[11] A. A. Mailybaev and A. P. Seyranian, On singularities of a boundary of the stability domain, SIAM J. Matrix Anal. Appl., 21 (2000), pp. 106-128.

[12] D. S. Schmidt, Transformations to versal normal form, in Computer Aided Proofs in Analysis, K. R. Meyer and D. S. Schmidt, eds., IMA Vol. Math. Appl. 28, Springer, New York, 1989, pp. 235-240.

[13] D. Schmidt, Versal normal form of the Hamiltonian function of the restricted problem of three bodies near $L_{4}^{*}$, J. Comput. Appl. Math., 52 (1994), pp. 155-176.

[14] L. Stolovitch, On the computation of a versal family of matrices, Numer. Algorithms, 4 (1993), pp. 25-56. 